\title{
INVESTIGACIONES
}

\section{Aprendizaje como participación durante el prácticum en un aula de Educación Infantil*}

\author{
Learning as participation during the practicum in a Pre-school Education Classroom \\ Aprender como participação durante o estágio profissionalizante \\ em uma sala de aula de Educação Infantil
}

\author{
Bárbara Toledo Fierro, ${ }^{a}$ Teresa Mauri Majós ${ }^{b}$
}

aUniversidad de Barcelona, España

Telf.: (34) 648763370. Correo electrónico: barbaratoledo.f@gmail.com

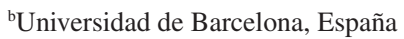

Telf.: (34) 933125823. Correo electrónico: teresamauri@ub.edu

\begin{abstract}
RESUMEN
El objetivo de este trabajo es analizar las formas de actividad conjunta que crean los implicados en las prácticas propias de una comunidad de aula, teniendo como finalidad conocer las oportunidades de aprendizaje y formación que le ofrecen al futuro maestro durante el prácticum. Adoptando una perspectiva sociocultural (Vigotsky, 1983), se explica el aprendizaje como participación en prácticas sociales y situadas (Lave \& Wenger, 1991). Mediante un estudio de caso, donde participan una maestra tutora, una maestra practicante y 18 alumnos/as durante 15 jornadas de aula, se analizan distintas formas de organización de la actividad conjunta (Coll, 1990) que soportan este aprendizaje. Los resultados muestran la estructura de participación que compone la actividad conjunta característica del aula y su variación a lo largo del transcurrir temporal, así como los grados de implicación de los participantes y, en consecuencia, las diferentes oportunidades de aprendizaje que le ofrece al estudiante de maestro.

Palabras clave: formación inicial del profesorado, prácticum, actividad conjunta, participación, comunidad de aula.
\end{abstract}

\section{ABSTRACT}

The aim of this work is to analyze the forms of joint activity that are created by those involved in practices of a classroom community, with the purpose of knowing the opportunities of learning and training that are offered to future teachers during their practicum. Adopting a sociocultural perspective (Vigotsky, 1983), learning is explained as participation in social and situated practices (Lave \& Wenger, 1991). Through a case study, where one tutorteacher, one student-teacher and 18 pupils participate during 15 school days, the different forms of organization of joint activity (Coll, 1990) that support this learning are analyzed. The results show the participation structure comprising the featured joint activity of the classroom and the temporal variations, as well as the degree of involvement of the participants and, consequently, the different learning opportunities offered to the student-teacher.

Key words: initial teacher training, practicum, joint activity, participation, classroom community.

Este trabajo se enmarca en la elaboración de una Tesis Doctoral financiada por CONICYT, Programa de Formación Humano Avanzado, Beca Chile 72110447, adjudicada a Bárbara Toledo Fierro. También se ha contado con el apoyo del en Interacción e Influencia Educativa (GRINTIE) al cual pertenecen los autores. 


\section{MARCO TEÓRICO}

Este trabajo se sitúa en el campo de investigación de la formación inicial del profesorado, centrándonos en la formación y el aprendizaje de los futuros maestros durante el prácticum. Específicamente, se identifica y analiza las oportunidades de aprendizaje de las que el estudiante de prácticum dispone por su participación en las prácticas particulares de una comunidad de aula.

En primer lugar, se aborda brevemente el concepto de prácticum en el marco de la formación inicial docente. A continuación, se presenta y justifica la noción de aprendizaje desde una perspectiva sociocultural, distinguiendo los conceptos clave para comprender nuestro abordaje teórico y metodológico. Finalmente, enmarcamos lo anterior en el contexto del aprendizaje de la enseñanza durante la estancia del estudiante en una comunidad de aula.

\subsection{EL PRÁCTICUM EN EL MARCO DE LA FORMACIÓN INICIAL DEL PROFESORADO}

En la literatura internacional existe consenso en relación a que la formación de los futuros maestros durante el período de prácticum representa un punto emblemático y de especial importancia en el marco de su formación profesional inicial, reconociéndose, en consecuencia, el valor de la experiencia práctica para el aprendizaje de la enseñanza (Latorre y Blanco, 2011; Le Cornu, 2008; Mattsson, Eilertsen y Rorisson, 2011; Mauri et al., 2015; Sorensen, 2014; Tejada, 2006; Walkington, 2005; Zabalza, 2011; Zeichner, 2011). Estos mismos autores también han puesto de relieve la necesidad de realizar mayores investigaciones sobre el prácticum, argumentando que es un proceso con un potencial formativo que no ha sido suficientemente explorado y que debería considerarse prioritario en la investigación educativa. Así, el estudio de este espacio de formación ha tomado especial relevancia en los últimos años, contando en la actualidad con un amplio volumen de investigaciones que, desde diversas perspectivas teóricas y de análisis, han profundizado en este período. Sin embargo, como sostiene Sorensen (2014), "systematic reviews of work in the field suggest a lack of detailed examination of the practices that are most effective in supporting student teachers' learning"' (p. 128). En consecuencia, el presente estudio atiende a esta necesidad de ejecutar análisis más profundos sobre los procesos y estructuras que tienen lugar e intervienen en el aprendizaje de los estudiantes de maestros durante el prácticum.

Entendemos por prácticum el período de tiempo durante el cual el estudiante de maestro se inserta en una institución profesional real, la escuela, y participa en la actividad habitual de un aula de clases. Los objetivos generales del practicum se orientan a ofrecer al estudiante las siguientes oportunidades: (i) observar y analizar tareas educativas y de enseñanza y aprendizaje que diariamente se llevan a cabo en un centro y un aula; e (ii) implicarse y participar en las mismas, diseñando y desarrollando propuestas de enseñanza que logren dar cuenta del grado de dominio que el estudiante posee de las competencias propias del docente. Durante el practicum, el estudiante desarrolla todas las actividades bajo la guía del maestro tutor responsable del aula, y del profesor tutor de la universidad que supervisa este período. Así, el prácticum se entiende, en primer lugar, enmarcado en

Traducción propia: "Revisiones sistemáticas de los trabajos en este campo sugieren la falta de un examen detallado de las prácticas que son más eficaces en el apoyo del aprendizaje de los estudiantes de maestro". 
un currículo específico de formación inicial, con propósitos y supuestos teóricos diversos según los diferentes planes de estudio de las universidades, y plasmado en un plan docente que diseña, estructura y encuadra la inmersión del futuro maestro en una escuela. En segundo lugar, el prácticum se considera como un período de actividad situada en un centro escolar particular, con características y cultura propia, y específicamente ubicado en un aula de clases. Los futuros maestros permanecen la mayor parte de las horas y desarrollan la mayoría de las tareas contempladas en el plan de prácticum en un aula de clases (Mattsson, et al., 2011; Tejada, 2006; Zabalza, 2011).

Autores como Edwards (2005) y otros (Mtika, Robson y Fitzpatrick, 2014; Rosaen y Florio-Ruane, 2008) plantean que la implicación de las escuelas en la formación docente de los futuros maestros se centra, en gran medida, en facilitarles la entrada a la institución y el contacto con el trabajo diario que se realiza en las aulas. Esta oferta se basa en el valor formativo que se atribuye a la posibilidad misma de estar presente en dicho trabajo. Sin embargo, en la práctica, esta oferta formativa resulta bastante inespecífica, ya que la responsabilidad de concretar en qué consiste dicha presencia o participación en dicho contexto, queda en manos del propio estudiante y de la relación que este establezca con el maestro tutor del aula.

Asimismo, algunos autores (Hagger y Hazel, 2006; Sorensen, 2014) indican que el patrón dominante del prácticum consiste en situar a un estudiante en una aula determinada con un maestro tutor concreto, permaneciendo aislados del resto de maestros del centro. Consecuentemente, parece ser que la escuela, entendida como unidad formativa global, no responde al papel de agente formador, es decir, el aprendizaje de los futuros maestros no es visto como una responsabilidad del centro en su conjunto, sino que se relega dicha tarea en manos exclusivamente de los maestros tutores.

Igualmente, los maestros tutores que asumen una función de guía del estudiante durante el prácticum fijan su compromiso con la formación centrándose principalmente en ofrecer al estudiante de maestro oportunidades de observación y experimentación puntual, sin que exista, en la mayoría de los casos, un plan de intervención intencional de formación. En definitiva, la intervención de los maestros tutores en la formación del estudiante de prácticum consiste, habitualmente, en facilitar momentos de observación no participante y/o un aprendizaje basado en el ensayo y error en tareas profesionales del aula (Hagger y Hazel, 2006).

En este trabajo se reconoce la importancia del carácter situado del prácticum, esto es, incrustado en un sistema social determinado. Asimismo, se considera que la mera presencia o exposición del estudiante de maestro a las actividades que se desarrollan en el aula, no garantiza la construcción de los aprendizajes esperados en este período. En consecuencia, el estudio que presentamos ubica el foco en este espacio concreto de formación durante el prácticum, el aula, específicamente en las prácticas particulares llevadas a cabo y la identificación de los aprendizajes que posibilita y/o limita a los estudiantes su participación en las actividades habituales de dicho contexto.

\subsection{APRENDIZAJE COMO PARTICIPACIÓN EN PRÁCTICAS PARTICULARES DE UNA COMUNIDAD}

Con la finalidad de caracterizar y comprender la idea del aprendizaje como participación, este estudio adopta una aproximación sociocultural (Lave \& Wenger, 1991; Vigotsky, 1983). Esta se basa en el supuesto principal de que la acción del individuo se encuentra 
situada, mediada y estrechamente vinculada a la actividad o sistema de actividad con otros, en el contexto en que se desarrolla. Esta aproximación extrae su marco básico, por una parte, de los trabajos de Vygotsky acerca de las funciones mentales superiores y la comprensión de estas a través del estudio de las relaciones sociales en las que el individuo existe, poniendo el foco en las herramientas culturalmente importantes en el marco de una actividad particular en interacción con otros (Vygotsky, 1983; Wertsch, 1993). Por otra parte, lo extrae de la teoría de la actividad propuesta por Leontiev, que establece que el individuo se apropia de los recursos culturales distribuidos en la comunidad mediante la participación a través de la acción en un sistema de actividad global (Leontiev, 1983; Wells, 2001).

En consecuencia con estos presupuestos básicos, pero con posterioridad a su planteamiento, se han desarrollado dos corrientes de estudio de la actividad. Una sitúa su foco de análisis en la explicación de los procesos individuales de internalización de los elementos culturales distribuidos en el sistema de actividad. La otra se centra en el carácter social de la actividad, focalizando su análisis en el aprendizaje como participación en prácticas particulares de un contexto, donde el aprendizaje viene dado a partir de la transformación de las formas de participación o actividad conjunta (Matusov, 1998).

Nuestro trabajo se enmarca en la segunda de estas corrientes: el modelo de aprendizaje como participación. Siguiendo a Matusov (1998), los planos social (interpsicológico) y personal (intrapsicológico) se constituyen mutuamente, existiendo el individuo en el flujo de las actividades socioculturales en las que participa con otros. La transformación de las formas de participación pone de relieve maneras de acción diferentes en un sistema de actividad, constituido en interacción con otros, por tanto, el aprendizaje vendría dado por las diversas formas de participar en una actividad sociocultural particular de una comunidad.

En definitiva, el aprendizaje es considerado un proceso sociocultural situado, cuya dirección, contenido y medios son definidos por la comunidad, específicamente, por las prácticas particulares en las que el sujeto se inserta, participa y contribuye en su construcción, siendo así un aspecto integral e inseparable de la práctica misma con otros en dicho contexto (Lave y Wenger, 1991; Rogoff, 1995; Wenger, 2001).

El estudio del aprendizaje como participación en prácticas particulares de una comunidad requiere la descripción y distinción de conceptos relevantes para su comprensión y análisis, los cuales desarrollaremos a continuación.

En primer lugar, de acuerdo con C. Coll, A. Bustos y A. Engel (2008), reconocemos la naturaleza compleja y multidisciplinaria del concepto de comunidad, el cual, de manera general, refiere a "un grupo de personas con características o intereses comunes, que pueden compartir, aunque no necesariamente, un objetivo específico, y que a menudo comparten un territorio o un espacio geográfico" (p. 301). Una comunidad concreta refiere a una configuración social donde las personas que forman parte de ella comparten convenciones y reglas explícitas e implícitas, confiriéndoles una identidad común construida conjuntamente, mediante actividades y prácticas, a través de la socialización, y la comunicación.

A su vez, Wenger (2001) asocia los términos de comunidad y práctica para proponer una definición de un tipo especial de comunidad: comunidad de práctica. Según este autor, una comunidad de práctica se caracteriza por la persecución de una empresa conjunta mediante la creación de prácticas particulares que entregan estructura y otorgan significado a lo que se realiza en dicha comunidad. La afiliación a una comunidad de práctica supone un compromiso mutuo por parte de los miembros, así como la negociación de esta empresa conjunta. Además, se desarrolla un repertorio compartido, es decir, un 
conjunto de recursos heterogéneos como rutinas, palabras, instrumentos, formas de hacer, etc., que obtienen coherencia al producirse en el curso de las prácticas particulares de dicha comunidad. Resumiendo, "las comunidades de práctica ponen el acento en comprender las comunidades como lugares de compromiso en la acción, en las relaciones interpersonales, en el conocimiento compartido y en la negociación" (C. Coll, A. Bustos y A. Engel, 2008: 302), representando, de esta manera, un espacio ventajoso de aprendizaje, pues ofrece el acceso a las competencias que allí se desarrollan (Wenger, 2001).

En segundo lugar, siguiendo a Schatzki $(2002,2010)$ y Smith (2011), en términos simples cuando hablamos de prácticas nos referimos al lugar donde los sujetos se reúnen, actúan e interactúan entre sí. Las prácticas corresponderían a descriptivos de los fenómenos sociales (Corradi, Gherardi y Verzelloni, 2010). Por tanto, no abogamos por una noción de prácticas como estática, inmóvil, preestablecida y ya disponible en una comunidad. Al contrario, las prácticas son espacios de actividad conjunta en constante cambio y movimiento, interdependientes entre sí, y caracterizadas por un lenguaje, un espacio-tiempo, y relaciones sociales particulares, más o menos distintivas, comunes y compartidas. A su vez, estos espacios de actividad conjunta, se organizan y formalizan en actividades características, que corresponden a eventos temporales producidos en un momento presente determinado y un lugar particular, componiendo el quehacer diario de los participantes en la comunidad (Mauri et al., 2013; Schatzki, 2010).

Las prácticas corresponden a formas que, si bien inicialmente pueden ser preconfiguradas en función del discurso, espacio-tiempo y relación social, no están en absoluto predeterminadas, pues se concretan y definen por la actividad misma de los participantes mientras estos las llevan a cabo (Kemmis et al., 2014). Esto es, las prácticas particulares de una comunidad tienen orígenes históricos, culturales y situacionales, y han evolucionado a través del tiempo con el objetivo de satisfacer las necesidades culturales del contexto donde ocurren, constituyéndose de manera particular en cada escenario. Por ende, una comunidad no debe ser entendida como un contenedor de prácticas predefinidas, sino que las prácticas de dicha comunidad adquieren diversas formas y, más en concreto, formas de actividad conjunta, en función de las condiciones particulares del lugar y momento en que se dan y de la actividad misma de los participantes (Edwards, Gilroy y Hartley, 2002; Sannino, Daniels y Gutiérrez, 2009).

En síntesis, los procesos que soportan el aprendizaje dan forma a las prácticas particulares de una comunidad. El aprendizaje corresponde a distintas formas de participación del individuo con otros en dichas prácticas particulares, y que suponen diferentes formas de interpretar y responder en la actividad que se lleva a cabo. Así, la comunidad aparece como un espacio interactivo donde se preconfiguran un conjunto de prácticas particulares que representan para los implicados diversas oportunidades de participación en las actividades en que se organizan, y variados grados de acción e intervención.

\subsection{EL PRÁCTICUM COMO PARTICIPACIÓN EN LAS PRÁCTICAS DE UNA COMUNIDAD DE AULA}

Korthagen (2010) y otros (Edwards y Protheroe, 2003; Kemmis, Edwards-Groves y Heikkinen, 2012; Kemmis et al., 2014), plantean que adquirir una visión del aprendizaje como participación en las prácticas particulares de una comunidad, posee un potencial considerable para comprender el aprendizaje profesional de futuros maestros, y el desarrollo de una formación docente basada en la escuela. Sin embargo, no se trata de una perspectiva 
simplificada a nociones de aprender haciendo, o la inclusión individual del estudiante de maestro en las prácticas de las escuelas. Mas bien, se propone considerar el aprendizaje de los estudiantes de prácticum como parte de un proceso participativo, mediante el cual el futuro maestro sea capaz de realizar interpretaciones cada vez más informadas de los eventos ocurridos en el aula, y desarrollar repertorios de respuestas cada vez más amplios, mientras actúa en las actividades que organizan la práctica particular del aula.

Conceptualizamos el aula como una comunidad donde ocurren prácticas particulares, organizadas en diversas actividades rutinarias implícitas y explícitas donde interactúan maestros/as y alumnos/as. Por tanto, el prácticum supone un período de formación donde se le ofrece al estudiante la oportunidad de participar, de diferentes maneras, en dichas prácticas particulares, tomando parte en las actividades diarias, que posibilitan y limitan el desarrollo de aprendizajes profesionales, preparándose así para participar en un futuro en situaciones semejantes.

Como en cualquier comunidad, las prácticas particulares de un aula se concretan y organizan en diversas actividades rutinarias, diseñadas intencional y principalmente para el logro de los objetivos curriculares correspondientes, en las que intervienen conjuntamente maestros/as y alumnos/as. Sin embargo, dichas prácticas no corresponden a estructuras sociales predefinidas que ordenan un aula determinada uniformemente. Al contrario, las prácticas particulares de un aula son construidas mediante interacciones cotidianas entre los participantes, y entre estos y los recursos materiales, en momentos concretos y bajo condiciones y circunstancias particulares de dicha comunidad de aula, representando asî elementos específicos del contexto (Kemmis et al., 2012, 2014).

Dicho lo anterior, para comprender el aprendizaje profesional del futuro maestro es necesario situar el foco en las actuaciones de los participantes en las prácticas particulares de la comunidad de aula y los diferentes caminos que recorren para participar con otros de tal o cual manera. Esta interacción representa la materia prima mediante la cual es posible acceder a las prácticas particulares del aula, cuyas distintas formas de participación representan, a su vez, las oportunidades de aprendizaje que ofrece dicho contexto a los futuros maestros. Kemmis et al. (2014) plantean que, como prácticas distintivas, las prácticas de un aula corresponden a una forma de actividad establecida socialmente, donde las actividades y actuaciones que la estructuran derivan de los objetivos curriculares de llegar a conocer y/o hacer por parte de los alumnos/as. Consecuentemente, para un estudiante que lleva a cabo su prácticum, el aprendizaje correspondería a un proceso de iniciación e incorporación en dichas prácticas particulares y la contribución a su caracterización mediante la actuación con otros en el desarrollo de las mismas, lo cual implica participar en las actividades características, bajo diversas condiciones y circunstancias; y mediante esta participación explorar las posibilidades y limitaciones presentes en la interacción y características de esa práctica, volviéndose así más hábiles en dichas interacciones con los demás y con los recursos de dicha comunidad.

Conocer las prácticas particulares, y las actividades que las estructuran, puestas a disposición para la interacción de un estudiante de prácticum y los demás miembros de una comunidad de aula, permite entonces analizar y comprender el repertorio de actuaciones situadas a las que tiene acceso el estudiante, así como las diferentes formas de implicación en dichas prácticas (Edwards et al., 2002; Kovalainen y Kumpulainen, 2007).

Sin embargo, las prácticas parecen ser invisibles y tácitas (Engeström, 2007), incluso para aquellos que actúan directamente en ellas. En este sentido, complementando el marco 
teórico expuesto, es necesario contar también con un marco metodológico y de análisis que permita capturar lo implícito con el objetivo de profundizar y comprender las prácticas particulares foco de interés.

\section{MARCO METODOLÓGICO}

Nuestro trabajo de investigación sigue una lógica interpretativa (Erickson, 1989), centrada en los significados y la vida social de los sujetos, reconociendo la necesidad de comprender de manera detallada las prácticas concretas objeto de investigación, y los significados locales de dichas prácticas para los propios sujetos involucrados. Se utiliza una metodología de estudio de caso (Stake, 2010; Yin, 2006) para analizar las particularidades y complejidades de un caso singular, mediante la observación y recogida de datos en el entorno natural.

Siguiendo criterios de selección, se escogió una estudiante de cuarto curso del Grado de Educación Infantil ${ }^{2}$ de una universidad catalana, a quien le correspondía realizar la asignatura de Prácticum II durante el primer trimestre del curso académico. De manera general, esta asignatura busca que el futuro maestro pruebe cómo se actúa profesionalmente, colaborando y participando en las dinámicas y proyectos del centro escolar; e interviniendo en el aula guiando procesos de desarrollo y aprendizaje ${ }^{3}$.

\subsection{SITUACIÓN DE OBSERVACIÓN}

Se registraron 15 Jornadas de Aula (JdA) del nivel de educación infantil P54, de un centro escolar público de la provincia de Tarragona (Cataluña, España), donde la estudiante seleccionada llevó a cabo el Prácticum II.

Las JdA estaban dirigidas a la enseñanza y aprendizaje de diversos contenidos curriculares del nivel educativo correspondiente. Exceptuando las intervenciones ejecutadas para la recogida de los datos, las JdA fueron planificadas y desarrolladas de manera natural.

La selección de las 15 JdA obedeció principalmente a dos criterios: (i) la representación del proceso de prácticum (5 jornadas correspondientes a la fase inicial, 5 jornadas correspondientes a la fase de desarrollo y 5 jornadas correspondientes a la fase final del prácticum); y (ii) la participación conjunta en el aula de la estudiante de prácticum, la maestra tutora ${ }^{5}$ y los/as alumnos/as.

En definitiva, los sujetos del caso correspondieron a 18 alumnos de entre 4 y 5 años de edad, una maestra tutora (MT), una maestra practicante (MP) y, cuando correspondió, una maestra de apoyo o una maestra de psicomotricidad.

\footnotetext{
El equivalente en Chile al grado de Educación Parvularia.

Información extraída del documento institucional universitario "Guía de Prácticum II".

El nivel de Educación Infantil en España corresponde a la etapa educativa que atiende a niños/as desde el nacimiento hasta los 6 años. Comprende 6 cursos que se organizan en dos ciclos: primer ciclo de 0 a 3 años; y segundo ciclo de 3 a 6 años de edad que contempla los cursos P3, P4 y P5.

5 En el sistema educativo español, el o la docente a cargo de cada grupo de niños de un aula es llamado Maestro/a Tutor/a (el equivalente a Profesor/a Jefe en el contexto chileno), cuya función general es hacer un seguimiento, tanto a nivel individual como de grupo, del proceso de aprendizaje de todo el alumnado, y coordinar la acción de otros maestros que intervienen en el grupo, vehicular la información a las familias y coordinarse con los servicios externos que intervienen en el grupo.
} 


\subsection{PROCEDIMIENTO DE RECOGIDA Y ANÁLISIS DE DATOS}

Las $15 \mathrm{JdA}$ se registraron mediante audio, vídeo y notas de campo, correspondiendo al total de horas que los/as alumnos/as permanecieron dentro del aula durante una mañana habitual de clases, formando un cuerpo de datos de aproximadamente 46 horas de registro de la actividad de aula. Además, los datos fueron complementados con entrevistas semiestructuradas iniciales y finales realizadas a MT y a MP, y la revisión de documentos institucionales.

En cuanto al modelo de análisis construido, este se concreta en dos niveles diferenciados, con finalidades específicas pero vinculadas entre sí. Aquí se presenta únicamente el primer nivel elaborado para analizar las formas de organización que toma la actividad conjunta (AC) entre MT, MP y alumnos/as, en torno a las actividades desarrolladas en el aula.

Siguiendo a Tharp et al. (2002) y Coll y colaboradores (Coll et al., 1995; C. Coll, J. Onrubia y T. Mauri, 2008; Colomina, Onrubia y Rochera, 2001), se considera que para conocer y comprender las prácticas particulares de un contexto educativo formal, como es el caso del aula, es necesario indagar en las características distintivas de este contexto y las formas en que se organiza la actividad conjunta entre los miembros que forman parte de él. Sin embargo, a diferencia de trabajos anteriores centrados en las formas de actividad conjunta asociada a la interiorización (Coll et al., 1995; C. Coll, J. Onrubia y T. Mauri, 2008), nuestro modelo de análisis sitúa el interés tanto en las formas que toma la actividad conjunta en sí misma como prácticas particulares del aula; así como en las posibilidades y limitaciones que suponen dichas formas para la participación.

En consecuencia, para cada JdA, se realizaron los siguientes pasos:

(a) Identificación de Segmentos de Interactividad (SI). Los SI corresponden a formas específicas de organización de AC en una JdA, dominados por una estructura de participación social y académica, y una unidad temática determinada; y caracterizados por la articulación de un patrón de actuaciones típicas desarrolladas por los participantes en la interacción. Representan una unidad analítica básica, inferida de los datos, que otorga una visión global de la estructura, distribución, articulación y evolución de la interactividad.

Se propone una doble caracterización para cada SI:

(i) Primer foco de caracterización. Centrado en la estructura de la actividad del aula, específicamente en las tareas que MT y MP (y maestra de apoyo o psicomotricidad si corresponde) realizan con los/as alumnos/as para dar cumplimiento al currículum escolar. Se trata de una aproximación diádica de la práctica del aula (actuaciones pertenecientes a las maestras por un lado, y a los/as alumnos/as por otro) que sienta sus bases en el modelo de análisis de la interactividad propuesto por Coll y colaboradores.

(ii) Segundo foco de caracterización. Encajado en el foco anterior, se centra en la forma en que cada maestra se implica en el desarrollo de las distintas formas de actividad conjunta descritas según el primer foco de caracterización. Se trata de una aproximación multivocal (considerando las actuaciones individuales de cada participante en interacción con los demás) focalizada en las formas de participación que MT, MP (y maestra de apoyo o psicomotricidad si corresponde) ponen de 
manifiesto al interactuar entre sí y con los/as alumnos/as en el marco de la actividad conjunta de primer foco ${ }^{6}$.

(b) Identificación de Actuaciones Típicas (AT) de cada SI. Las AT corresponden a acciones interactivas multimodales realizadas por los participantes con un significado determinado en el SI en que aparecen, dando cuenta del tipo de implicación de cada participante en la actividad conjunta y de la interrelación entre estos.

(c) Elaboración de Mapas de Interactividad (MI). Los MI son representaciones gráficas de la organización de los SI en cada jornada dando cuenta de la estructura que toma la actividad conjunta y su evolución en el tiempo. Estas representaciones consideran la simultaneidad temporal de algunos SI y los elementos considerados para caracterizarlos.

\section{RESULTADOS}

En este apartado se presentan, sucintamente, algunos resultados generales correspondientes al análisis de las $15 \mathrm{JdA}$ donde la futura maestra desarrolló su período de prácticum, así como ciertos resultados más específicos del análisis de datos seleccionados del corpus más amplio, que sirven como ejemplo ilustrativo y permiten al lector capturar y profundizar en el trabajo llevado a cabo; lo anterior debido a la limitación de espacio que dificulta la presentación de un mayor número de resultados obtenidos.

En primer lugar, a partir del análisis de la totalidad de los datos, se identificaron y caracterizaron veintiún formas distintas de organización de la actividad conjunta llevadas a cabo por los participantes, presentadas y descritas brevemente en la Tabla 1, y que corresponden a las prácticas particulares de la comunidad de aula estudiada.

Tabla 1. Prácticas particulares de la comunidad de aula

1. Acogida de los/as niños/as (AN). Organización de la actividad conjunta en torno al encuentro de familiares y niños/as que llegan al aula, y la/s maestra/s, con el objetivo de acoger a los/as niños/as y prepararlos para comenzar la jornada.

2. Inicio de la jornada de aula (IJA). Organización de la actividad conjunta en torno a situar a los/as niños/as, reencontrarse como grupo, y marcar un punto de partida para el desarrollo del resto de la jornada.

3. Aportación de información que anticipa la planificación de la jornada o semana $(A I A P)$. Organización de la actividad conjunta en torno a la presentación de información relativa a acontecimientos futuros, distribución organizativa de la jornada y/o la semana, anticipando a los/as niños/as acerca de lo que sucederá y deben hacer.

Este segundo foco de caracterización fue construido a partir de los antecedentes teóricos respecto del aprendizaje como participación y de investigaciones como las de Campbell (2012) y Fillietaz, Trebert y Rémery (2012). 


\begin{tabular}{|c|c|}
\hline & $\begin{array}{l}\text { Identificación de asistentes, fecha y tiempo (IAFT). Organización de la actividad } \\
\text { conjunta en torno a la ejecución de tareas de rutina de pase de lista y de actualización } \\
\text { de fecha y tiempo. }\end{array}$ \\
\hline 5. & $\begin{array}{l}\text { Petición de identificación y representación de cantidades y cifras numéricas (PIRC). } \\
\text { Organización de la actividad conjunta en torno a la ejecución de tareas de representación } \\
\text { de cifras con las manos, y/o identificación de cifras representadas en tarjetas. }\end{array}$ \\
\hline & $\begin{array}{l}\text { Evaluación de cálculo mental individual (ECM). Organización de la actividad } \\
\text { conjunta en torno a la evaluación de resolución de problemas de cálculo mental. }\end{array}$ \\
\hline 7. & $\begin{array}{l}\text { Compartir experiencias, hechos y/o conocimientos }(C E H C) \text {. Organización de la } \\
\text { actividad conjunta en torno a debatir en gran grupo, experiencias, acontecimientos y/o } \\
\text { hechos mediante intercambios conversacionales entre la/s maestra/s y los/as niños/as. }\end{array}$ \\
\hline 8. & $\begin{array}{l}\text { Transición. Organización de la actividad conjunta en torno a períodos de paso de una } \\
\text { actividad a otra. Existen tres subtipos de actividad conjunta de transición: }\end{array}$ \\
\hline & $\begin{array}{l}\text { 8.1. Transición con actividad delegada (TAD). Organización de la actividad conjunta } \\
\text { en torno a gestionar la diversidad de ritmos de finalización de una tarea, mediante } \\
\text { la realización de otras actividades mientras el resto del grupo completa la tarea } \\
\text { iniciada. }\end{array}$ \\
\hline & $\begin{array}{l}\text { 8.2. Transición con actividad acompañada (TAA). Organización de la actividad } \\
\text { conjunta en torno a la utilización del tiempo de espera del grupo clase en una } \\
\text { actividad complementaria, mientras el/la niño/a encargado/a finaliza la tarea } \\
\text { anterior. }\end{array}$ \\
\hline & $\begin{array}{l}\text { 8.3. Transición con traslado }(T T) \text {. Organización de la actividad conjunta en torno a } \\
\text { preparar y regular los desplazamientos del grupo clase fuera del aula y viceversa. }\end{array}$ \\
\hline & $\begin{array}{l}\text { Presentación de la tarea planificada (PTP). Organización de la actividad conjunta en } \\
\text { torno a la organización y presentación de una tarea planificada con contenido } \\
\text { específico. }\end{array}$ \\
\hline & $\begin{array}{l}\text { Trabajo en gran grupo }(T G G) \text {. Organización de la actividad conjunta en torno a la } \\
\text { ejecución conjunta, en el marco del gran grupo, de la tarea planificada, mientras la/s } \\
\text { maestra/s observa, guía, corrige y valora. }\end{array}$ \\
\hline 11. & $\begin{array}{l}\text { Trabajo en la pizarra }(T P) \text {. Organización de la actividad conjunta en torno a la } \\
\text { ejecución individual o en pequeño grupo de grafía en la pizarra alrededor de un } \\
\text { contenido, mientras la/s maestra/s observa, guía, corrige y valora. }\end{array}$ \\
\hline & $\begin{array}{l}\text { Trabajo individual en mesas y valoración (TIMV). Organización de la actividad } \\
\text { conjunta en torno a la ejecución individual, en el marco del gran grupo, de la tarea } \\
\text { planificada, mientras la/s maestra/s observa, guía, corrige y valora. }\end{array}$ \\
\hline
\end{tabular}




\begin{tabular}{|l|l|}
\hline 13. Trabajo individual en plástica y valoración (TIPV). Organización de la actividad \\
conjunta en torno a la ejecución individual, en el marco del gran grupo, de la tarea en \\
plástica, mientras la/s maestra/s observa, guía, corrige y valora.
\end{tabular}

Para cada una de estas formas de organización de la actividad conjunta se identificó detalladamente el patrón de actuaciones típicas que los participantes llevan a cabo, y que definen y limitan cada una de las actividades, considerando, sin embargo, que estas actuaciones sufren variaciones de frecuencia y forma de aparición a lo largo de las jornadas de aula. En la Tabla 2 se presenta, a modo de ejemplo ilustrativo, el patrón de actuaciones típicas de dos forma de organización de la actividad conjunta: Acogida de los/as niños/as y Presentación de la tarea planificada. Ambas resultaron ser significativas en términos de frecuencia y regularidad de aparición a lo largo de las 15 JdA. 
Tabla 2. Patrón de Actuaciones Típicas del Segmento de Interactividad Acogida de los/as niños/as y Presentación de la tarea Planificada

\begin{tabular}{|c|c|}
\hline PARTICIPANTES & SI DE ACOGIDA DE LOS/AS NIÑOS/AS \\
\hline Maestra/s & $\begin{array}{ll}\text { - } & \text { Recibe/saluda a los/as niño/as } \\
\text { - } & \text { Saluda/conversa con familiares } \\
\text { - } & \text { Recibe documentos/materiales de mano de familiares o niños/as } \\
\text { - } & \text { Formula/recuerda las consignas a los/as niños/as } \\
\text { - } & \text { Establece/recuerda normas, límites y reglas del aula a los/as niños/as } \\
\text { - } & \text { Otorga asistencia a los/as niño/as sobre diversas necesidades } \\
\text { - } & \text { Mantiene charla social con los/as niño/as } \\
\text { - } & \text { Organiza, prepara y/o presenta los recursos materiales }\end{array}$ \\
\hline Niños/as & $\begin{array}{l}\text { - } \\
\text { - } \\
\text { - } \\
\text { - } \\
\text { - }\end{array}$ \\
\hline PARTICIPANTES & SI DE PRESENTACION DE LA TAREA PLANIFICADA \\
\hline Maestra/s & $\begin{array}{l}\text { - } \text { Formula una propuesta de inicio de la tarea a los/as niños/as } \\
\text { - Organiza, prepara, presenta y/o reparte los recursos materiales } \\
\text { - } \text { Establece/recuerda normas, límites y reglas del aula a los/as niños/as } \\
\text { - } \text { Formula, repite y/o amplía las instrucciones de la tarea a los/as niños/as } \\
\text { - } \text { Formula requerimientos en relación con un ámbito de contenido } \\
\text { - } \text { específico a uno/a o más/as niños/as } \\
\text { - } \text { más niños/as } \\
\text { - } \quad \text { Sesponde, comenta y/o valora las intervenciones de uno/a o más niños/as } \\
\text { - Otorga seguimiento y ayuda a uno/a o más niños/as }\end{array}$ \\
\hline Niños/as & $\begin{array}{l}\text { - Atienden las explicaciones de la/s maestra/s } \\
\text { - Demandan clarificaciones sobre la tarea } \\
\text { - Plantean preguntas, dudas o comentarios sobre la tarea } \\
\text { - Responden los requerimientos realizados por la/s maestra/s } \\
\text { - } \quad \text { Siguen las instrucciones dadas por la/s maestra/s }\end{array}$ \\
\hline
\end{tabular}

En segundo lugar, encajadas en las formas de organización de la actividad conjunta presentadas, se encontraron nueve formas distintas de participación, descritas brevemente en la Tabla 3, que reflejan el grado de implicación que manifiestan la maestra tutora y maestra practicante (y maestra de apoyo o maestra de psicomotricidad cuando correspondió) en dichas prácticas particulares de la comunidad de aula. 
Tabla 3. Formas de participación construidas por las participantes en interacción en las prácticas particulares de la comunidad de aula

1. Observación con intervención puntual (OIP). La actividad conjunta de MT y MP está definida por la toma de control primordial de la actividad por parte de MT, mientras MP adopta una postura de observación externa, ocupando un papel de 'testigo' de la actividad que se está desarrollando con los/as niños/as.

2. Co-participación con apoyo (CpA). La actividad conjunta de MT y MP está definida por la toma de control primordial de la actividad por parte de MT, mientras MP toma parte de la actividad ejecutando una o más partes de la tarea, pero sin involucrarse globalmente en ella.

3. Co-participación con implicación (CpI). La actividad conjunta de MT y MP está definida por la toma de control primordial de la actividad por parte de MT, mientras MP toma parte activa en el desarrollo global de la tarea, centrándose principalmente en la supervisión del trabajo de los/as niños/as y la ayuda individual a estos/as.

4. Co-participación con delegación $(C p D)$. La actividad conjunta de MT y MP está definida por la toma de control primordial de la actividad por parte de MT, mientras MP, sin involucrarse en la actividad que se está desarrollando con el gran grupo, ejecuta paralelamente una tarea que ha sido delegada implícita o explícitamente por MT.

5. Co-participación con distribución (CpDt). La actividad conjunta de MT y MP está definida por la ejecución paralela de dos tareas de aprendizaje distintas llevadas a cabo en pequeño grupo, haciéndose cargo cada maestra de uno de ellos.

6. Co-participación con traspaso con implicación (CpTI). La actividad conjunta de MT y MP está definida por la toma del control primordial de la actividad por parte de MP, mientras MT observa e interviene complementando las acciones de MP, principalmente en la regulación de la participación de los/as niños/as y/o la aportación de información.

7. Co-participación con traspaso con apoyo (CpTA). La actividad conjunta de MT y MP está definida por la toma del control primordial de la actividad por parte de MP, mientras MT intercala momentos de ejecución de tareas paralelas y momentos de observación de la actividad que se está desarrollando, interviniendo puntualmente.

8. Co-participación con traspaso sin intervención (CPTsI). La actividad conjunta de MT y MP está definida por la toma del control primordial de la actividad por parte de MP, mientras MT sale del aula y/o ejecuta una tarea paralela sin observar ni intervenir en la actividad que se está desarrollando con los/as niños/as.

9. Presencia sin intervención o ausencia (NI). La actividad conjunta de MT y MP está definida por la toma de control primordial de la actividad por parte de MT, mientras MP sale del aula y/o ejecuta una tarea paralela sin observar ni intervenir en la actividad que se está desarrollando con los/as niños/as. 
De igual manera, para cada una de estas formas de participación se describió detalladamente el patrón de actuaciones típicas de cada uno de los participantes, en el marco de la forma de actividad conjunta que desarrollan conjuntamente. En la Tabla 4 se presenta, a modo de ejemplo ilustrativo, el patrón de actuaciones típicas de la forma de participación Co-participación con implicación en el marco de la actividad conjunta Trabajo individual en mesas y valoración. Esta combinación corresponde a una dinámica significativa en términos de frecuencia y regularidad de aparición a lo largo de las $15 \mathrm{JdA}$ analizadas.

Tabla 4. Patrón de Actuaciones Típicas de la forma de participación Co-participación con implicación en la práctica Trabajo individual en mesas y valoración

\section{PARTICIPANTES SI DE TRABAJO INDIVIDUAL EN MESAS Y VALORACIÓN}

Formula una propuesta de inicio de la tarea a los/as niños/as.

Otorga seguimiento y/o ayuda a los/as niños/as en el desarrollo de la tarea.

Maestra Tutora

Establece/recuerda normas, límites y reglas del aula a los/as niños/as.

Formula, repite y/o amplia las instrucciones de la tarea a los/as niños/as.

Corrige y/o valora el resultado individual de la tarea.

Formula una propuesta de cierre de la tarea a los/as niños/as.

\begin{tabular}{ll}
\hline & Observa las actuaciones llevadas a cabo por la maestra tutora y los/as \\
Mañostra Practicante & Otorga en el desaimiento y/o ayuda a los/as niños/as en el desarrollo de la tarea. \\
& Formula, repite y/o amplia las instrucciones de la tarea a los/as niños/as
\end{tabular}

$\begin{array}{ll} & \text { Ejecutan individualmente la tarea } \\ \text { Niños/as } & \text { Demandan ayuda y/o consultan dudas } \\ & \text { Muestran el resultado individual de la tarea }\end{array}$

La identificación y descripción detallada, tanto de las distintas formas de organización de la actividad conjunta de los participantes — prácticas particulares de la comunidad del aula—, así como de las diferentes formas de participación construidas en la interacción grado de implicación de cada maestra-, permite describir las JdA en función del cruce de ambos focos. Así, encontramos un total de noventa y siete combinaciones distintas entre las prácticas particulares del aula con su correspondiente grado de implicación por parte de las maestras, distribuidas diferenciadamente durante el desarrollo del prácticum.

A modo de ejemplo ilustrativo, la Figura 1 muestra la distribución, articulación y tiempo (en minutos) de la actividad conjunta a lo largo de $3 \mathrm{JdA}$ mediante este cruce combinado - mapas de interactividad- Los colores corresponden a las prácticas particulares de la comunidad de aula (Tabla 1) y la trama corresponde a la forma de participación (Tabla 3). Las $3 \mathrm{JdA}$ graficadas son representativas de las fases de inicio, desarrollo y final del prácticum.

A través de estos mapas es posible observar las diferencias y regularidades en la organización de las actividades y formas de participación disponibles. De las veintiuna 
Estudios Pedagógicos XLII, $\mathrm{N}^{\circ}$ 2: 343-363, 2016 APRENDIZAJE COMO PARTICIPACIÓN DURANTE EL PRÁCTICUM EN UN AULA DE EDUCACIÓN INFANTIL

Figura 1. Mapas de Interactividad de tres Jornadas de Aula del proceso de prácticum

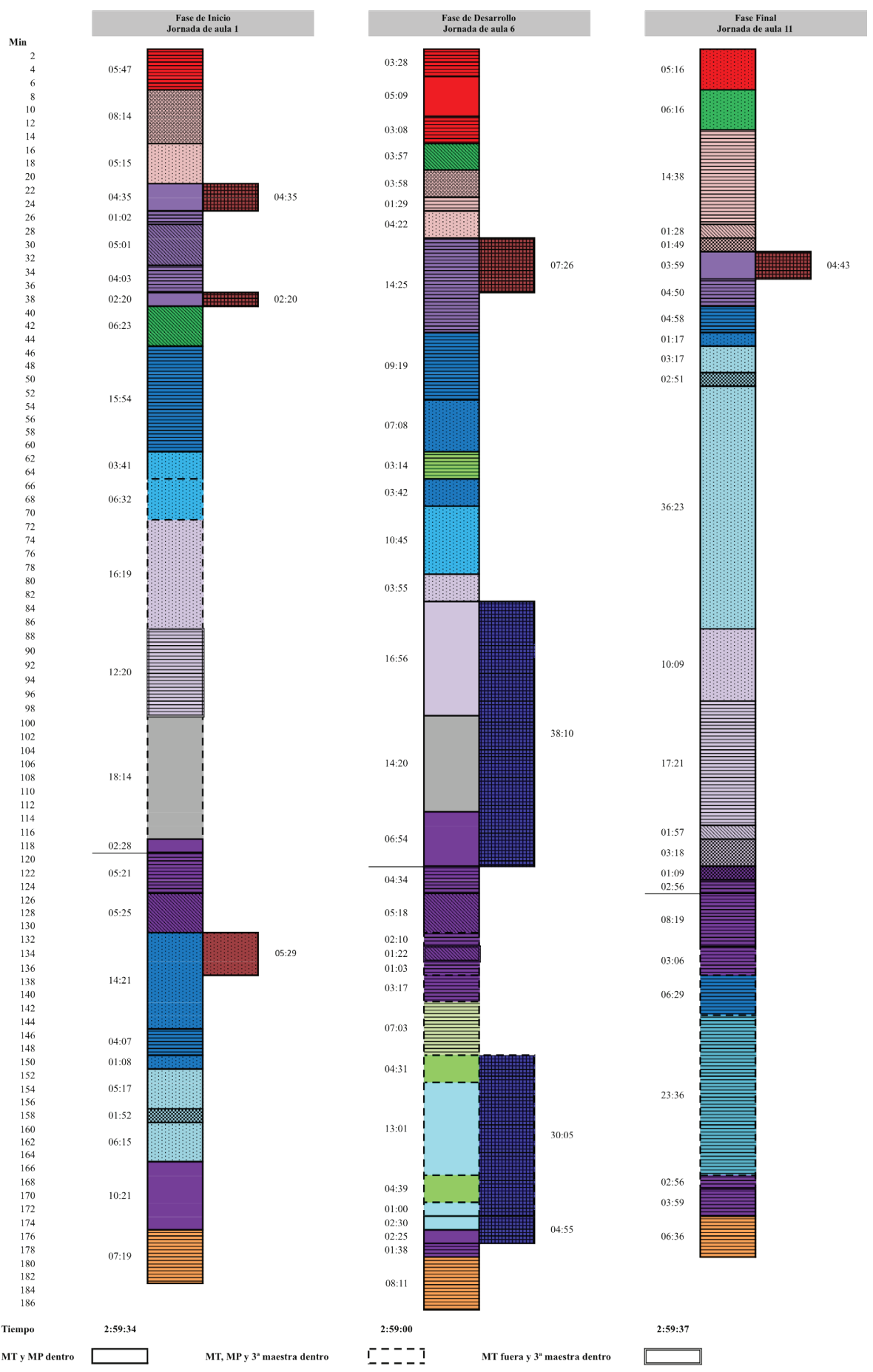


actividades características del aula identificadas, diecisiete de ellas aparecen distribuidas en las 3 jornadas de aula graficadas: diez están presentes en las 3 jornadas, dos aparecen en 2 jornadas y 5 aparecen solo en una. Por su parte, de las nueve formas de participación identificadas, siete de ellas aparecen distribuidas en las 3 jornadas graficadas: cinco están presentes en las 3 jornadas y dos aparecen en 2 de las jornadas.

Esta combinación de ambos focos de caracterización de la actividad conjunta a lo largo del período de prácticum entrega, por un lado, información acerca de la secuencia de aparición y distribución de las actividades características de la comunidad del aula en las jornadas, secuencia que, a pesar de no presentar igual aparición y/o distribución en todas las jornadas de aula, se muestra medianamente estable a lo largo del eje temporal, estableciéndose una rutina de actividades características de la comunidad de aula que toma como punto de referencia el horario escolar, y que varía en función de la planificación diaria.

Por otro lado, a partir del cruce de ambos focos es posible observar también la forma de participación o grado de implicación que cada maestra tiene, momento a momento, en el desarrollo de cada actividad característica, transparentándose las actuaciones que son llevadas a cabo y que están disponibles para ser ejecutadas por los participantes durante la interacción.

\section{DISCUSIÓN Y CONCLUSIONES}

En este último apartado, se resumen los hallazgos clave que surgieron del análisis de 15 jornadas de una comunidad de aula de Educación Infantil donde participaron conjuntamente alumnos/as, maestra tutora y maestra practicante, mientras esta última realizaba su período de prácticum.

Considerando que durante este período de formación el aprendizaje profesional del futuro maestro corresponde a un proceso de participación en las prácticas particulares de la comunidad de aula en la que se inserta, la comprensión de las oportunidades de aprendizaje disponibles para la estudiante de prácticum depende de:

(i) la caracterización detallada de las prácticas particulares del aula que construyeron los participantes. Por tanto, encontramos veintiún tipos diferentes, que en función de su aparición y distribución en las jornadas de aula, revelan indicadores estables de las formas en que se organiza la actividad conjunta al interior de esta comunidad, cada una de ellas caracterizada, a su vez, por un patrón de actuaciones típicas disponibles para ser ejecutadas o no por parte de la futura maestra. Esta red de prácticas particulares sirve como matriz donde situar.

(ii) la descripción de las distintas formas de participación manifestadas por las maestras en el marco de la interacción con los demás participantes en las prácticas particulares identificadas, con especial interés en cómo la estudiante de prácticum se involucra, contribuye y hace suyo este patrón de actuaciones típicas que estructuran cada práctica del aula. Por ende, los resultados muestran nueve formas de participación distinta y variables en términos de evolución y trayectoria a través de las jornadas, descritas en función de las formas de actuaciones individuales de los participantes en interacción con los demás.

(iii) la representación gráfica y descripción de la organización de las prácticas particulares de la comunidad del aula en cada jornada y su evolución a través del tiempo. 
Al igual que Kovalainen y Kumpalainen (2007), se considera que las prácticas particulares de la comunidad de aula estudiada son definidas y construidas socialmente a través de las actuaciones de los participantes. Esto supuso implicaciones teóricas y metodológicas que nos han llevado a desarrollar un procedimiento de recogida y análisis de datos que permitió trasparentar dichas prácticas, haciendo visibles su forma y función, y el conjunto de actuaciones típicas a las que las maestras y los/as alumnos/as podían o no suscribirse. Coincidimos con autores como Clarke (2004) y Young y Miller (2004), quienes proponen las prácticas del aula como actividades colaborativas, construidas a partir de la interacción entre maestras/os y alumnos/as, por lo que solo pueden ser entendidas mediante un análisis que considere las aportaciones de todos los participantes involucrados a la actividad conjunta.

A su vez, y de acuerdo con Kemmis et al. (2014), en el caso de las prácticas particulares de un contexto, estas dependen además de las circunstancias específicas del centro escolar en general, y del aula en particular, es decir, estas prácticas particulares se desarrollan en respuesta a las necesidades, condiciones y oportunidades del aula específica. De este modo, desde un punto de vista del aprendizaje profesional del estudiante de prácticum, este tiene la oportunidad de participar de diferentes maneras en estas prácticas - y no otras- que están dirigidas principalmente a dar cumplimiento al currículo escolar del nivel educativo correspondiente.

En consecuencia, y de acuerdo con lo expuesto al principio de este trabajo, la estudiante de prácticum, mientras se encuentra dentro de la comunidad del aula, es considerada, más que una aprendiz a quien brindarle apoyo y guía, como una visitante temporal a quien se le da acceso al aula y mientras se encuentre allí puede colaborar con ciertas tareas de baja complejidad. Lo anterior moldea de manera importante las oportunidades de acción disponible para la estudiante de maestra y las propias prácticas construidas en interacción con los demás. Por consiguiente, a pesar de que a esta estudiante se le solicita explícitamente, a través del plan de prácticum, actuar en las actividades habituales llevadas a cabo dentro del aula, no se le otorga ningún derecho de impacto, cambio y/o innovación en dichas actividades, salvo en casos en que la actividad en sí corresponde a una exigida expresamente desde la universidad. Se confirma así lo planteado por algunos autores respecto a que el objetivo primordial de las prácticas llevadas a cabo en una aula donde se recibe a un estudiante de prácticum, corresponde al aprendizaje y avance curricular de los/ as niños/as, objetivo que no cambia cuando la estudiante de maestra realiza su estancia de formación dentro de ella (Edwards et al., 2002; Toledo y Mauri, 2014, 2015).

En la misma línea, tomando las ideas propuestas por Engestöm (2001) acerca del aprendizaje en un sistema de actividad, y coincidiendo con Campbell (2012), nuestros resultados muestran que, viendo el aula como sistema de actividad, el objeto que orienta la actividad del aula es el aprendizaje de los contenidos por parte de los/as alumnos/as. De tal manera, el aula representa un sistema de actividad intencionalmente diseñado para que los niños/as aprendan los contenidos curriculares esperados.

Ahora, en el caso del prácticum llevado a cabo por un estudiante de maestro dentro de un aula, el objeto buscado que orienta la actividad es el aprendizaje de dicho estudiante, pues el propósito de la asignatura de prácticum, de manera general, es que los futuros maestros aprendan sobre la enseñanza en dicha aula (Campbell, 2012; Douglas, 2010). Sin embargo, a pesar de que Engeström (2001) plantea que cuando un sistema de actividad adopta un nuevo elemento desde el exterior, como podría ser el caso del estudiante de maestro durante 
el prácticum, esto frecuentemente conduce a la renegociación del objeto de la actividad; en el caso de nuestra investigación esto no se da. A partir de la descripción de las prácticas particulares de la comunidad de aula estudiada, es posible ver la primacía del objeto de actividad orientado al aprendizaje y avance curricular de los niños/as, objeto que no entra en negociación con el objeto orientado hacia el aprendizaje del estudiante de prácticum.

En definitiva, cuando un estudiante de maestro realiza su prácticum dentro de una comunidad de aula, el objeto de la actividad, y los demás elementos relacionales, se mantienen tal y como se encontraban antes que entrara este nuevo participante. Eventualmente, como propone Campbell (2012), las herramientas que median la actividad de los alumnos/as, son susceptibles de transformarse en herramientas de mediación del aprendizaje de los futuros maestros y viceversa, como en los casos de las actividades desarrolladas como parte del plan de prácticum.

Teniendo en cuenta lo anterior, resulta claro que las necesidades que satisfacen las prácticas particulares del aula regulan deliberadamente las formas de participación de maestras y alumnos/as en dichas prácticas, y, en consecuencia, el tipo de oportunidades de aprendizaje disponibles para la futura maestra. Asimismo, las formas de participación que lleva a cabo la estudiante de prácticum en dichas prácticas se vuelven clave para comprender qué y cómo aprenden los futuros maestros durante el prácticum.

Sin embargo, resulta necesario señalar que nuestros resultados cuestionan las propuestas de algunos autores, como Lave y Wenger (1991), que conciben el proceso de participación como algo lineal y predeterminado, que sigue una trayectoria desde la participación periférica a la plena participación. Al contrario, coincidimos con aquellos autores que plantean la construcción de distintas dinámicas de interacción, que no siguen un trayecto preestablecido, sino que dependen de la practica particular, y más específicamente, de las actividades que se están llevando a cabo momento a momento (Fillietaz et al., 2012; Kovalainen y Kumpulainen, 2007; Mauri et al., 2013). Por tanto, son las prácticas particulares del aula, construidas por la actividad conjunta de los participantes, las que proporcionan innumerables y distintas oportunidades de acción e implicación, y por ende distintas oportunidades de aprendizaje, para un futuro maestro (Edwards et al., 2002).

En resumen, sin querer ser críticos, el objetivo de este trabajo es el de identificar y comprender formas en que las prácticas particulares de una comunidad de aula donde una estudiante realiza su prácticum, pueden resultar beneficiosas y productivas, o perjudiciales e ineficientes para el aprendizaje profesional. Una vez que identificamos tales prácticas y la forma en que los participantes se implican en ellas, quizás sea posible pensar en cómo podrían ser transformadas y mejoradas, considerando que la transformación de una práctica requiere la transformación de las interacciones que la produce. En este sentido, coincidimos con Kemmis et al. (2014), al plantear que la ampliación y desprivatización de las prácticas particulares del aula resulta clave para el aprendizaje de los futuros maestros. Como ya se ha dicho, históricamente los maestros actúan a puerta cerrada dentro de sus aulas, situación que no cambia cuando ingresa un estudiante de prácticum. Desprivatizar las prácticas sería una buena manera de ampliar también la responsabilidad en relación a la formación inicial docente en los centros escolares, y no limitarla exclusivamente a un maestro y sus alumnos/as detrás de cuatro paredes. Lo anterior no solo supone la apertura de espacios físicos, sino de nuevos espacios de interacción social lo cual implicaría la creación de nuevas prácticas que permitan nuevas formas de aprendizaje profesional a los futuros maestros (Mauri et al., 2015). 
Finalmente, considerando los hallazgos presentados, y tomándolos como referente, el siguiente paso contemplado será profundizar en la comprensión de cuándo, cómo y por qué ocurre el paso de una forma de participación a otra, analizando la construcción progresiva de cada una de estas formas de participación, desde su inicio hasta su estabilización, y los elementos interactivos verbales y no verbales involucrados, en el marco de la actividad conjunta entre maestra tutora, maestra practicante y alumnos/as.

\section{REFERENCIAS BIBLIOGRÁFICAS}

Campbell, S.S. (2012). Taking them into the field: Mathematics teacher candidate learning about equity-oriented teaching practices in a mediated field experience (Unpublished doctoral thesis). University of Washington, Seattle.

Clarke, D. (2004). Patterns of Participation in the Mathematics Classroom. In M.J. Hoines \& A.B. Fuglestad (Eds.), Proceedings of the 28th Conference of the International Group for the Psychology of Mathematics Education (pp. 231-238). Bergen: Bergen University College.

Coll, C. (1990). Un marco de referencia psicológico para la educación escolar: la concepción constructivista del aprendizaje y la enseñanza. En C. Coll, J. Palacios y A. Marchesi (Eds.), Desarrollo psicológico y educación. II. Psicología de la educación (pp. 435-453). Madrid: Alianza.

Coll, C., Bustos, A., \& Engel, A. (2008). Las comunidades virtuales de aprendizaje. En C. Coll y C. Monereo (Eds.), Psicología de la educación virtual (pp. 299-320). Madrid: Morata.

Coll, C., Colomina, R., Onrubia, J., \& Rochera, M. (1995). Actividad conjunta y habla: Una aproximación a los mecanismos de influencia educativa. En P. Fernández y M. Melero (Comps.), La interacción social en contextos educativos (pp. 193-326). Madrid: Siglo XXI.

Coll, C., Onrubia, J., \& Mauri, T. (2008). Ayudar a aprender en contextos educativos: el ejercicio de la influencia educativa y el análisis de la enseñanza. Revista de Educación, 346, 33-70.

Colomina, R., Onrubia, J., \& Rochera, M. (2001). Interactividad, mecanismos de influencia educativa y construcción del conocimiento en el aula. En C. Coll, J. Palacios y A. Marchesi (Eds.), Desarrollo Psicológico y Educación. Vol. II. Psicología de la Educación Escolar (pp. 437-458). Madrid: Alianza.

Corradi, G., Gherardi, S., \& Verzelloni, L. (2010). Through the practice lens: Where is the bandwagon of practice-based studies heading? Management Learning, 4(3), 265-283.

Douglas, A.S. (2010). What and how do student teachers learn from working in different social situations of development in the same school? In V. Ellis, A. Edwards, \& P. Smagorinsky (Eds.), Cultural-Historical Perspectives on Teacher Education and Development: Learning Teaching (pp. 30-44). Abingdon: Routledge.

Edwards, A. (2005). Let's get beyond community and practice: the many meanings of learning by participating. Curriculum Journal, 16(1), 49-65.

Edwards, A., Gilroy, P., \& Hartley, D. (2002). Rethinking teacher education: collaborative responses to uncertainty. Londres: Routledge.

Edwards, A., \& Protheroe, L. (2003). Learning to see in classrooms: what are student teachers learning about teaching and learning while learning to teach in schools? British Educational Research Journal, 29(2), 227-242.

Engeström, Y. (2001). Expansive learning at work: toward an activity theoretical reconceptualization. Journal of Education and Work, 14(1), 133-156.

Engeström, Y. (2007). From communities of practice to mycorrhizae. In J. Hughes, N. Jewson, \& L. Unwin (Eds.), Communities of practice: Critical perspectives (pp. 41-54). Londres: Routledge.

Erickson, F. (1989). Métodos cualitativos de investigación sobre la enseñanza. En M. Wittrock (Ed.), 
La investigación de la enseñanza II. Métodos cualitativos y de observación (pp. 195-301). Barcelona: Paidós Ibérica, S.A.

Filliettaz, L., Trébert, D., \& Rémery, V. (2012). Relation tutorale et configuration de participation à l'interaction: le cas de la formation professionnelle des éducatrices et éducateurs de l'enfance. Document présenté à 2ème colloque international de didactique professionnelle, Apprentissage et développement professionnel. 7-8 Juin, Université de Nantes, Nantes, Francia.

Hagger, H., \& Hazel, H. (2006). Learning teaching from teachers: realizing the potential of schoolbased teacher education. Buckingham: Open University Press.

Kemmis, S., Edwards-Groves, C., \& Heikkinen, H.L.T. (2012). Teacher induction as practice. Paper presented at Symposium Research on practices of teacher induction at the European Conference on Educational Research, 18-21 September, University of Cádiz, Cádiz, España.

Kemmis, S., Wilkinson, J., Edwards-Groves, C., Hardy, I., Grootenboer, P., \& Bristol, L. (2014). Changing Practices, Changing Education. Singapore: Springer.

Korthagen, F.A.J. (2010). Situated learning theory and the pedagogy of teacher education: towards and integrative view of teacher behavior and teacher learning. Teaching and Teacher Education, 26(1), 98-106.

Kovalainen, M., \& Kumpalainen, K. (2007). The social construction of participation in an elementary classroom community. International Journal of Educational Research, 46(3-4), 141-158.

Latorre, M.J., \& Blanco, F. J. (2011). El prácticum como espacio de aprendizaje profesional para docentes en formación. REDU Revista de Docencia Universitaria, 9(2), 35-54.

Lave, J., \& Wenger, E. (1991). Situated Learning: Legitimate Peripheral Participation. Cambridge: Cambridge University Press.

Le Cornu, R. (2008). The changing role of the 'student teacher' in professional experience. Paper presented at Australian Association for Research in Education Conference, 30 November-4 December, Queensland University, Brisbane, Australia.

Leontiev, A. (1983). El desarrollo del psiquismo. Madrid: Akal.

Mattsson, M., Eilertsen, T., \& Rorisson, D. (2011). What is practice in teacher education? In M. Mattsson, T. Eilersten, \& D. Rorrisson (Eds.), A Practicum Turn in Teacher Education (pp. 1-15). Roterdam/Bostom/Taipei: Sense Publishers Pedagogy.

Matusov, E. (1998). When solo activity is not privileged: Participation and internalization models of development. Human Development, 41(5-6), 326-349.

Mtika, P., Robson, D., \& Fitzpatrick, R. (2014). Joint observation of student teaching and related tripartite dialogue during field experience: Partner perspectives. Teaching and Teacher Education, 39, 66 -76.

Mauri, T., Clarà, M., Colomina, R., \& Onrubia, J. (2015). Naturaleza de la interacción en procesos de reflexión conjunta sobre situaciones de la práctica por estudiantes de maestro. Papeles de Trabajo sobre Cultura, Educación y Desarrollo Humano, 11(2), 105-109.

Mauri, T., Clarà, M., Ginesta, A., \& Colomina, R. (2013). La contribución al aprendizaje en el lugar de trabajo de los equipos docentes universitarios. Un estudio exploratorio. Infancia y Aprendizaje, 36(3), 341-360.

Rogoff, B. (1995). Observing sociocultural activity on three planes: Participatory appropriation, guided participation and apprenticeship. In J. Wertsch, P. del Río, \& A. Álvarez (Eds.), Sociocultural studies of mind (pp. 139-164). Cambridge: Cambridge University Press.

Rosaen, C., \& Florio-Ruane, S. (2008). The metaphors by which we teach: Experience, metaphor, and culture in teacher education. In M. Cochran-Smith, S. Feiman-Nemser, J. McIntyre, \& K. Demers (Eds.), Handbook of research on teacher education: Enduring questions in changing contexts (3rd ed., pp. 706-731). New York: Routledge, Taylor \& Francis.

Sannino, A., Daniels, H., \& Gutiérrez, K.D. (2009). Activity Theory between Historical Engagement and Future-Making Practice. In A. Sannino, H. Daniels, \& K. D. Gutiérrez (Eds.), Learning and Expanding with Activity Theory (pp. 1-16). New York: Cambridge University Press. 
Schatzki, T.R. (2002). The site of the social: A philosophical account of the constitution of social life and change. Pennsylvania: University of Pennsylvania Press.

Schatzki, T.R. (2010). Materiality and Social Life. Nature and Culture, 5(2), 123-149.

Smith, M.K. (2011). What is praxis? The encyclopadia of informal education. Recuperado desde http://www.infed.org/biblio/b-praxis.htm

Sorensen, P. (2014). Collaboration, dialogue and expansive learning: the use of paired and multiple placements in the school prácticum. Teaching and Teacher Education, 44, 128-137.

Stake, R. (2010). Investigación con estudio de casos. Madrid: Morata.

Tharp, R. G., Estrada, P., Stoll, D., Stephanie, Y., \& Lois, A. (2002). Transformar la enseñanza: excelencia, equidad, inclusión y armonía en las aulas y en las escuelas. Barcelona: Paidós.

Tejada, J. (2006). El Prácticum. Actas de las Jornadas sobre el futuro Grado de Pedagogía (pp. 1226). Barcelona: Edición Técnica Digital Universidad de Barcelona.

Toledo, B., \& Mauri, T. (2014). Construcción social de las prácticas de una comunidad de aula de educación infantil: Análisis de la actividad conjunta de la maestra tutora, maestra practicante y alumnos/as. En M.A. Flores, C. Coutinho, \& J.A. Lencastre, (Org.), Atas do congresso Formação e trabalho docente na sociedade da aprendizagem. Organizado no âmbito da International Study Association on Teachers and Teaching (ISATT) e do Centro de Investigação em Estudos da Criança (CIEC) (pp. 489-500). Braga: CIEC, UMinho.

Toledo, B., \& Mauri, T. (2015). Dinámicas de participación en una comunidad de aula: Oportunidades de aprendizaje del futuro maestro durante el período de prácticum. Papeles de Trabajo sobre Cultura, Educación y Desarrollo Humano, 11(2), 121-127.

Vigotsky, L. (1983). Pensamiento y Lenguaje. Teoría del desarrollo cultural de las funciones psíquicas. Buenos Aires: Pléyade.

Walkington, J. (2005). Becoming a teacher: encouraging development of teacher identity through reflective practice. Asia-Pacific Journal of Teacher Education, 33(1), 53-64.

Wells, G. (2001). Indagación dialógica. Hacia una teoría y una práctica socioculturales de la educación. Barcelona: Paidós.

Wenger, E. (2001). Comunidades de práctica. Aprendizaje, significado e identidad. Barcelona: Paidós.

Wertsch, J. (1993). Voces de la mente: un enfoque sociocultural para el estudio de la acción mediada. Madrid: Visor Distribuciones.

Yin, R. (2006). Case Study Methods. In J. Green, G. Camilli, \& P. Elmore (Eds.), Handbook of complementary methods in education research (pp. 111-122). Mahwah: Lawrence Erlbaum Associates.

Young, R., \& Miller, E. (2004). Learning as changing participation: discourse roles in ESL writing conferences. Modern Language Journal, 88(4), 519- 535.

Zabalza, M.A. (2011). El prácticum en la formación universitaria: estado de la cuestión. Revista de Educación, 354, 21-43.

Zeichner, K. (2011). Rethinking the connections between campus based courses and field experiences in college and university based teacher education. In J. Millwater, L. Ehrich, \& D. Beutel (Eds.), Practical Experiences in Professional Education: A transdisciplinary approach (pp. 37-60). Brisbane: Post Pressed. 
